\title{
Malaria vector control at a crossroads: public health entomology and the drive to elimination
}

\author{
Abraham P. Mnzava ${ }^{a, *}$, Michael B. Macdonald ${ }^{a}$, Tessa B. Knox ${ }^{a}$, Emmanuel A. Temu ${ }^{a, b, c}$ and Clive J. Shiff \\ ${ }^{a}$ Vector Control Unit, Global Malaria Programme, World Health Organization, Geneva 1211, Switzerland; ${ }^{b}$ Swiss Tropical and Public Health \\ Institute, Basel 4003, Switzerland; 'University of Basel, Basel 4003, Switzerland; ${ }^{d}$ The W. Harry Feinstone Department of Molecular \\ Microbiology and Immunology, Johns Hopkins Bloomberg School of Public Health, Baltimore, MD 21205, USA \\ *Corresponding author: Tel: +41 22791 3613; Fax: +41 22794 824; E-mail: mnzavaa@who.int
}

Received 6 March 2014; revised 2 June 2014; accepted 2 June 2014

\begin{abstract}
Vector control has been at the core of successful malaria control. However, a dearth of field-oriented vector biologists threatens to undermine global reductions in malaria burden. Skilled cadres are needed to manage insecticide resistance, to maintain coverage with current interventions, to develop new paradigms for tackling 'residual' transmission and to target interventions as transmission becomes increasingly heterogeneous. Recognising this human resource crisis, in September 2013, WHO Global Malaria Programme issued guidance for capacity building in entomology and vector control, including recommendations for countries and implementing partners. Ministries were urged to develop long-range strategic plans for building human resources for public health entomology and vector control (including skills in epidemiology, geographic information systems, operational research and programme management) and to set in place the requisite professional posts and career opportunities. Capacity building and national ownership in all partner projects and a clear exit strategy to sustain human and technical resources after project completion were emphasised. Implementing partners were urged to support global and regional efforts to enhance public health entomology capacity. While the challenges inherent in such capacity building are great, so too are the opportunities to establish the next generation of public health entomologists that will enable programmes to continue on the path to malaria elimination.
\end{abstract}

Keywords: Capacity building, Entomology, Epidemiology, Malaria, National malaria control programme, Vector control

\section{Introduction}

Malaria control is at a critical juncture. Progress in global malaria control over the past decade was largely attained through rapid scale-up of vector control as well as diagnosis and treatment with effective combination therapy. The wide-scale deployment of long lasting insecticidal nets (LLINs) and indoor residual spraying (IRS) alone accounted for $>60 \%$ of the total estimated US $\$ 2.5$ billion of international and domestic funding for malaria control. Significant results were achieved. According to WHO World Malaria Report 2013, between 2000 and 2012, estimated global malaria mortality fell by $45 \%$ in all age groups and by $51 \%$ in children under 5 years. Nevertheless, between 2011 and 2012, the pace of decrease in estimated malaria mortality slowed. ${ }^{1}$ It is evident that malaria elimination in many settings might not be achieved, nor even current gains sustained, without adapting to the changing threats to and opportunities for reducing transmission. Given the critical role of vector control and the magnitude of investments in vector control commodities and their deployment, surprisingly little has been done to build the local infrastructure and skills needed to sustain coverage, monitor impact and improve the efficiency of these interventions. Development of an adequate cadre of individuals with entomological skills and the corresponding infrastructure to support their activities has been especially neglected. Insectaries and entomological laboratories are often run-down or absent. Collaboration between national malaria control programmes (NMCP) and national universities and training institutions is often weak. Entomologists have few opportunities for training and generally poor prospects for employment once trained. ${ }^{2}$ Yet the future is promising: new opportunities exist to expand the role of those involved in vector control to perform as public health entomologists and contribute towards malaria elimination.

\section{Enhance entomological capacity to eliminate malaria}

In September 2013, WHO issued guidance to countries and partners on the need to build capacity in entomology and vector control, recognising that global malaria control efforts are entering a new era that requires improved entomological surveillance 
for more efficient and well-monitored vector control investments. NMCPs are required to adapt to the emerging threat of insecticide resistance, ${ }^{3}$ to outdoor and early vector biting ${ }^{4}$ and to shifting transmission patterns brought about through changing ecologies owing to such factors as deforestation, agricultural and infrastructural development, and urbanisation. Programmes need more robust surveillance and a well-defined implementation research agenda in order to ensure cost-effective use of resources as they move from control towards elimination. ${ }^{5-7}$ This will require specific training in programme management, surveillance and epidemiology, in addition to vector biology and entomology.

Entomological surveillance and a clear understanding of local transmission ecology are central to reducing transmission receptivity and preventing re-establishment of malaria where it has been eliminated. ${ }^{8}$ Challenges presented by residual transmission of malaria by vector populations not targeted by LLINs and IRS, such as early morning, early evening or outdoor biters, may be considerable. ${ }^{6,9}$ Indeed, the contexts of malaria vector control and the role of the vector biologist are changing, reflecting innovation in tools both for control interventions and entomological monitoring, including use of mobile technologies, communications, information management, climate data and mapping for gaining greater efficiencies. Investment in entomological monitoring and surveillance makes good business sense. In this era of increasing insecticide resistance and concerns for sustained funding, programmes must build capacities to select and apply the right vector control methods at the right times and in the right places.

\section{Develop infrastructure to generate evidence base}

Basic entomological laboratories are needed to support mosquito collection, rearing, morphological identification and bioassays. At the national level, there is a minimum requirement for an insectary to maintain an insecticide-susceptible Anopheles colony for use in IRS and LLIN bioefficacy evaluations and as a reference for insecticide resistance assays. An entomological reference laboratory supporting molecular identification of vector species is important where morphologically identical sibling species may be sympatric and can provide valuable information on resistance mechanisms, sporozoite rates and blood meal composition. These facilities require specific equipment, supplies and expertise to remain functional; this may best be handled on a centralised basis.

However, such facilities are seldom established and managed by NMCPS. In some countries these facilities exist in national universities or research and training institutes. ${ }^{10}$ In such cases, it may be possible for the NMCP to initiate a contractual arrangement for the provision of insectary or reference laboratory services, but they must be aware that the priorities of a research institute may be upstream research rather than routine entomological monitoring and surveillance. As such, these agreements should serve as an interim solution while NMCPs develop the necessary insectary and laboratory capacity within the programme instead of having to rely on other institutions.

Vector control programmes also need capacity for basic implementation research to examine the local situation in terms of the entomological and epidemiological impact of interventions, the impact of insecticide resistance, the optimal timing and frequency of IRS applications, and the potential contribution of residual transmission of malaria. ${ }^{6}$ Co-ordination with national and, where needed, external universities or research institutions is crucial; the NMCP should work with these partners to develop a research agenda to respond to programme needs. This active involvement of the NMCP in co-ordination and implementation of the research will promote data sharing (and circumvent the withholding of data for publication purposes) and make timely use of information to support policy and procurement decisions.

\section{Leverage new technologies and skills to enable adaptability}

In the past, an understanding of geographical and ecological factors was central to developing strategies to reduce malaria transmission. ${ }^{11}$ Known as 'species sanitation,' this required precise identification of the vectors and knowledge of their ecological requirements in order to modify the environment and eliminate their role in malaria transmission. While our modern-day approaches include LLINs and IRS, a fundamental understanding of ecology remains essential, especially in heterogeneous and changing environments. It is clear that NMCPs cannot sustain a one-size-fits-all approach to vector control. In the move towards elimination, it becomes critically important that resources are targeted effectively. ${ }^{12}$

Opportunities exist to refine strategies based on mobile communication technologies and geographic information systems. Data that 20 years ago were being entered by hand and processed on main-frame computers can now be largely collected, collated and analysed via a smart phone and a laptop. Rapid diagnostic tests for malaria have introduced a new dimension in monitoring malaria in real time; the rural health centre is now a source of critical information that can be accessed frequently and expressed in terms of case incidence rate. ${ }^{12,13}$ Many mosquito abatement programmes in developed countries, as well as some NMCPS, have established simple and robust information management and mapping tools that incorporate both epidemiological and entomological data.

Such systems can effectively pinpoint foci where transmission is occurring and enable the NMCP to devise immediate and targeted vector control interventions. In areas where transmission is declining, this could help sustain the elimination process in a cost-effective manner, ${ }^{14}$ but appropriately trained personnel are required. Control programmes must take advantage of this technology revolution and build capacity in skills and resources to utilise surveillance data, including incorporation of costing information and temporal-spatial analyses. Use of these tools will ultimately lead to more cost-effective implementation and greater sustainability of control interventions.

\section{Train and mentor public health entomologists for sustainability}

Examples of ongoing initiatives to support entomological capacity building of NMCPs and the research institutes that support their activities are presented in Box 1 . Along with training and mentoring for national entomology and vector control services, ${ }^{15,16}$ it is critical to provide career structures for these and other scientific 
Box 1. Examples of entomological capacity-building opportunities for national malaria and vector control programmes

\begin{abstract}
African Network on Vector Resistance (ANVR): This network supports member countries in the collection and publication of information on insecticide resistance, prepares frameworks and protocols for resistance surveillance and management and provides an avenue for communication and mentoring. It has also fostered collaboration between control programmes and research institutes at national and international levels and has organised training in entomology in many countries with the support of WHO.
\end{abstract}

\section{The International Masters Degree in Entomology (IME):}

Established in 2006 and jointly run by the Institute for Research and Development (Benin), Montpellier University (France),

Abomey-Calavi University (Benin) and the Entomological Research Centre of Cotonou (Benin), this course covers systematics, biology and ecology of vectors of medical interest and the epidemiology and control of vector-borne diseases. In its first 6 years, a total of 91 students from 24 countries graduated from this course; 38 graduates have since undertaken a $\mathrm{PhD}$ and 26 are now working in national research institutions or in national vector control programmes.

\section{Master of Science (MSc) Degree in Medical Entomology and} Vector Control: Establishment of a regional MSc course in entomology and vector control was supported by WHO Eastern Mediterranean Regional Office in collaboration with the Blue Nile Institute of Gezira University (Sudan), London School of Hygiene and Tropical Medicine (UK), Liverpool School of Tropical Medicine (UK) and Witwatersrand University (South Africa). Over 80 people from 12 countries of the African and Eastern Mediterranean WHO regions have been trained over the past 3 years. The course curriculum was also adapted for national training efforts in Iran and Pakistan. ${ }^{15}$

\section{Training Module on Malaria Control: Entomology and Vector} Control: WHO published a core structure for the integrated vector management (IVM) training curriculum in $2012^{16}$ and a training module on malaria entomology and vector control in 2013. ${ }^{15}$ The latter was designed primarily to help health personnel involved in the planning, implementing and evaluation of malaria vector control activities but also for those responsible for training such personnel. It reflects current vector control policies and guidelines.

Post-Graduate Degree Course in Public Health Entomology: Organized by the Vector Control Research Centre (WHO collaborating centre) affiliated to the Pondicherry University, Pondicherry (India).

Six month Diploma Course in Applied Parasitology and Entomology: Organized at the Institute of Medical Research (WHO collaborating centre), Kuala Lumpur (Malaysia).

personnel. Commonly, within a hierarchy dominated by the medical profession, career opportunities and leadership positions for the public health entomologist or vector biologist may be limited. In medically-oriented institutions such as most ministries of health, where career advancement is dependent on medical qualifications rather than public health or biology qualifications, it can be difficult to integrate career opportunities for scientists into the system, but it can be done. Without a concerted effort to address this issue, eager graduates wanting to pursue a career in vectorborne diseases will continue to compare the life-long opportunities of a public health control programme with that of research institutions, and choose the latter. To obviate this, attractive career opportunities for public health biologists need to be developed. As employment will be in a Ministry of Health, which is programmed to accommodate medical, technical and administrative personnel, some appropriate consideration must include careers for scientific graduate personnel. This may even require a revision of service conditions to create a civil service compatible with scientists as well as medical personnel. ${ }^{17}$

While the public health entomologist needs to understand much more than entomology, medical professionals should also understand the basics of applied entomology, including the key role played in the control of vector-borne diseases, with vector control consistently forming the core of public health efforts against malaria. In other words, the curative professions would benefit from courses in public health entomology and its interface with epidemiology and programme management as a central component of their training. This multidisciplinary approach will foster an appreciation of the respective contribution of, and need for, both curative and public health cadres.

It is clear that public health entomologists are needed in ministries of health, and some countries have succeeded in establishing effective public health programmes with scientists in leadership positions. National programmes in Myanmar, South Africa, Sudan and Zimbabwe have clearly defined cadres with different training and job descriptions, ranging from the national senior entomologist, to entomologists at the state level, assistant entomologists, and finally to the foundation of mosquito collectors. Integrating epidemiology and data management skills into such a structure may be feasible and attractive to NMCPS. Some national programmes rotate staff responsibilities on a seasonal basis in an attempt to maximise output from limited employees; mosquito collectors or assistant entomologists may be focused on entomological monitoring for only certain months of the year, alternating perhaps as environmental health officers during the remainder of the year. Whatever the exact civil service structure and retention schemes that operate in a country, as part of an overall national capacity building plan there is a need to critically address the job descriptions and the career structures for technicians, mid-level entomology and vector control staff and national-level public health entomologists.

The recommendations provided by $\mathrm{WHO}$ include specific actions to be undertaken at the national level with support from WHO and partners, to inventory and revise post descriptions, establish career development opportunities and strengthen a system whereby a young, newly-qualified technician or vector biologist will pursue a career in a field in which their skills are so critically needed.

\section{Increase WHO and partner support to countries}

In the past, WHO played an important role in training entomologists and vector control experts to support the work of control programmes in disease-endemic countries. In the mid-20th century, 
WHO was the authoritative institution that helped harmonise efforts across borders, provided technical advice upon request and tracked diseases based on periodic reports from member states. Their staff were also directly involved in supporting countries in the implementation of vector control interventions, including monitoring and evaluation. In 1982 WHO had 24 entomologists in country offices, 7 across all 6 regional offices and 7 at WHO headquarters. With those numbers, there was already considered to be a human resource crisis in the area of entomology and vector control. ${ }^{18}$ However, there are currently only 16 PhD entomologists across all of WHO (7 in WHO headquarters, 4 across the regional offices and 5 across the country offices), and only 4 of these work solely on malaria vector control ( 3 in WHO headquarters and 1 in WHO Regional Office for Africa). With the current level of investment in malaria vector control interventions, WHO's human capacity and structure is currently inadequate to support both its key normative role as a source of strategic and policy guidance and its ability to directly support the realisation of in-country implementation. WHO has embarked upon a broader process of reform to better meet the expectations of its member states and partners in addressing agreed global health priorities.

Today there are significantly more partners supporting malaria control implementation than in the past. This is due at least in part to the actions of the Roll Back Malaria Partnership. However, partner focus has too often been on the implementation of short-term projects rather than long-term programmatic development to ensure sustainability. There is little emphasis on capacity strengthening and preparation for hand-over of management responsibility to national programmes at the conclusion of projects. Donor emphasis on capacity building and on its co-ordination at the national level is essential, especially considering the changing landscape of malaria control towards elimination. Clear strategies and a long-term vision are needed for building and sustaining a new generation of public health entomologists and vector biologists, epidemiologists and administrators to manage national activities long after the donor departs.

Supporting this long-term vision for sustained and effective vector control is a concerted and co-ordinated effort to build a network of training and mentoring opportunities for public health entomologists and vector control specialists. Under the continued leadership of WHO, key areas for partner support should include: the development and revision of curricula and support for regional courses that can be adapted for national training institutions; identification and, where relevant, strengthening of the institutions to host proposed regional training courses using revised curricula; and fellowships for both short- and long-term training of prospective entomology professionals.

There is an obligation on the part of the partnerships to shoulder the responsibility, because without a competent and permanent scientific base to the national programme, implementation will be suboptimal. In the words of the public health advisor Socrates Litsios, 'To control malaria under current conditions requires an international response, one that builds national public health capacities capable of fully supporting local initiatives. ${ }^{19}$

\section{Conclusions}

There is a critical need to enhance institutional, infrastructural and human resources and to leverage partnerships for improved and expanded entomological capacity to support optimised malaria programming. This is crucial throughout all malaria control and elimination phases in order to guide the most cost-effective and locally-adaptive deployment of vector control resources. Investments in human resources and systems are critically needed to respond to the threats of insecticide resistance; to improve efficiencies to maintain vector control coverage; to develop innovative tools and strategies to address transmission beyond traditional IRS and LLINs; and, to develop an evidencebased, rational decision-making process to optimise the deployment of resources. There are opportunities not just for training, but also for continued mentoring and the establishment of clear job descriptions and career structures for cadres from the basic technician and mosquito collector to the national vector control programme manager.

Enhancing the potential for, and interest in, careers in vector biology and control is essential. This can be done by expanding the role of the traditional medical entomologist by augmenting with knowledge in public health, programme management, technology application especially geographic information systems and data management, and providing a role in the design, deployment and monitoring of new interventions. The role must be expanded from a medical to a public health entomologist, from a technician to a leader, implementer and problem-solver, with a broader range of technical and management skills that can provide a fulfilling life-long career- and an essential contribution to national health and development.

\section{Recommendations}

The guidance note issued by WHO in September 2013 contained key recommendations on entomology and vector control capacity building directed at countries and their partners. ${ }^{20}$

Malaria-endemic countries were encouraged to establish intersectoral co-ordination mechanisms led by the Ministry of Health to develop long-range strategic plans for building human resources and systems for public health entomology and vector control. This process should include collaboration between ministries, universities and research institutions to conduct training needs assessments and to develop multidisciplinary curricula that incorporate subjects critical to effective vector control planning and implementation. Implementation research agendas should be clearly defined to guide development of the requisite technical capacity for surveillance and control activities. Professional positions and career development structures at national and subnational levels should be established or revised. Countries must ensure that sufficient resources for human and infrastructure capacity are properly budgeted for in NMCP strategic plans.

Partners are urged to include a clear component of capacity building in all support to countries. This could include technical or financial support for curricula development, training tailored to vector control programme needs, mentoring and establishment or enhancement of insectary or laboratory facilities as well as support for implementation research in line with the control programme needs. A core consideration will be to promote country ownership of activities and outcomes and to ensure sustained technical vector control resources after partner support ends. 
WHO will contribute through collaborations with countries and their partners to devise and implement a capacity-building strategic plan, including conducting a needs assessments, developing locally-appropriate training curricula and producing and disseminating training materials. WHO may also provide training, technical advice and mentoring for optimal implementation of appropriate control strategies. The Organization will also support advocacy for the appropriate development of public health entomology professionals.

While the recommendations outlined above may be met with enthusiasm by those currently engaged in vector control and by the broader malaria community, their uptake and implementation will require active and sustained commitment. This review and the resulting recommendations were developed with significant input from WHO Technical Expert Group. However, political commitment will be needed in order for the appropriate strategies and resources to be availed for translating these recommendations to action at country level. Given the many partners involved in malaria control and elimination both at the global and national levels, enacting these recommendations may be costly and challenging in the short-term. We are, however, confident that this can be achieved with the concerted political commitment and effective engagement of relevant partners, with ministries of health driving and co-ordinating the process.

Authors' contributions: APM, MBM and CJS conceived the initial report entitled Capacity Building in Entomology and Vector Control as submitted to WHO Malaria Policy Advisory Committee in September 2013; APM, MBM, TBK and EAT consolidated the content into manuscript format. All authors read and approved the final manuscript. APM is the guarantor of the paper.

Acknowledgments: Members of WHO Malaria Vector Control Technical Expert Group are acknowledged for their valuable input on the initial report.

Funding: This work was supported by WHO.

Competing interests: None declared.

Ethical approval: Not required.

\section{References}

1 WHO. World Malaria Report 2013. Geneva: World Health Organization; 2013.

2 Mnzava A, Williams J, Bos R et al. Implementation of integrated vector management for disease vector control in the Eastern Mediterranean: where are we and where are we going? East Mediterr Health J 2011;17:453-9.

3 WHO. Global Plan for Insecticide Resistance Management in Malaria Vectors. Geneva: World Health Organization; 2012.
4 Mutuku FM, King CH, Mungai P et al. Impact of insecticide-treated bed nets on malaria transmission indices on the south coast of Kenya. Malar J 2011;10:356.

5 Karunaweera ND, Galappaththy GN, Wirth DF. On the road to eliminate malaria in Sri Lanka: lessons from history, challenges, gaps in knowledge and research needs. Malar J 2014;13:59.

6 Durnez L, Coosemans M. Residual transmission of malaria: an old issue for new approaches. In: Manguin S (editor). Anopheles Mosquitoesnew insights into malaria vectors. InTech 2013;672-704.

7 Bayoh MN, Mathias DK, Odiere MR et al. Anopheles gambiae: historical population decline associated with regional distribution of insecticide-treated bed nets in western Nyanza Province, Kenya. Malar J 2010;9:62.

8 Ferguson HM, Dornhaus A, Beeche A et al. Ecology: a prerequisite for malaria elimination and eradication. PLoS Med 2010;7:e1000303.

9 Geissbuhler Y, Chaki PC, Emidi B. Interdependence of domestic malaria prevention measures and mosquito-human interactions in urban Dar es Salaam, Tanzania. Malar J 2007;19:126.

10 Mutero CM. Directory of African Institutions with Existing Capacity for Training in Integrated Vector Management (IVM). RTI-International/ USAID Support for Capacity-building in Integrated Vector Management (IVM Task Order II); 2010. http://www.rbm.who.int/ partnership/wg/wg_itn/docs/ws8/DirectoryIVMinstitutionsAfrica.pdf [accessed 11 June 2014].

11 Bradley DJ. Watson, Swellengrebel and species sanitation: environmental and ecological aspects. Parassitologia 1994;36: 137-47.

12 Stresman GH, Kamanga A, Moono P et al. A method of active case detection to target reservoirs of asymptomatic malaria and gametocyte carriers in a rural area in Southern Province, Zambia. Malar J 2010;9:265.

13 Shiff CJ, Stoyanov C, Choobwe C et al. Measuring malaria by passive case detection: a new perspective based on Zambian experience. Malar J 2013;12:120.

14 Rathor HR, Mnzava A, Bile KM et al. Launching the first postgraduate diploma in medical entomology and disease vector control in Pakistan. East Mediterr Health J 2010;16(Suppl):S76-81.

15 WHO. Training Module on Malaria Control: entomology and vector control. Geneva: World Health Organization; 2013.

16 WHO. Core Structure for Training Curricula on Integrated Vector Management. Geneva: World Health Organization; 2012.

17 Paterson TT. Report of the Commission of Inquiry into the Organization and Development of the Southern Rhodesia Public Services. Salisbury, Southern Rhodesia: Government Printer; 1963.

18 US National Research Council. Manpower Needs and Career Opportunities in the Field Aspects of Vector Biology: report of a Workshop, Washington D.C. and Berkeley Springs, West Virginia: September 29-October 2, 1982. Washington, DC: Board on Science and Technology for International Development, Office of International Affairs, National Research Council; 1983.

19 Litsios S. The Tomorrow of Malaria. Wellington, New Zealand: Pacific Press; 1997.

20 WHO. Guidance Note on Capacity Building in Malaria Entomology and Vector Control. Geneva: World Health Organization; 2013. 